J. Mol. Biol. (1975) 91, 421-438

\title{
Dual Control of Lysogeny by Bacteriophage P22 : An Antirepressor Locus and its Controlling Elements
}

\author{
Mrron Levine, Susan Trumsdeli $\dagger$ \\ T. Ramakrishnan $\ddagger$ and Morley J. Bronson $\S$ \\ Department of Human Genetics \\ University of Michigan \\ Ann Arbor, Mich. 48104, U.S.A. \\ (Received 1 July 1974, and in revised form 30 September 1974)

\begin{abstract}
Two distantly linked clusters of genes on the Salmonella typhimurium phage P22 ehromosome are involved in the control of lysogeny and superinfection immunity. One cluster consists of genes $c 1, c 2$, and $c 3$, which are primarily responsible for the establishment and maintenance of lysogeny. It has been proposed that the second cluster consists of three loci which are responsible for the synthesis and control of an antirepressor substance which overcomes the repression mediated by the $c 2$ gene product. This paper reports the isolation of mutants in a locus designated "ant" having characteristics expected of antirepressor mutants. Evidence is presented that the other loci in this second immunity region, mnt and virA, control the expression of the ant gene as repressor and promoter/operator, respectively. The interactions of these three loci with each other and with the other immunity region are discussed.
\end{abstract}

\section{Introduction}

Temperate phage are characterized by the ability to repress their own replication and to coexist stably with the host bacterium as prophage. Temperate phages $\lambda$ and P22 accomplish this repression by means of a repressor protein which acts to prevent expression of most prophage genes and also to prevent expression of genes by homoimmune superfecting phage. In phage $\lambda$, repression of prophage and superinfecting phage is achieved by the binding of $c I$ gene product, the repressor, to two operator sites on either side of the $c \mathrm{I}$ gene, thereby preventing transcription of essential early genes (see review by Ptashne, 1971). Phage P22 is similar to $\lambda$ in the arrangement of genes on the chromosome and in some aspects of the regulation of gene expression. Genetic studies indicate that the $c 2$ gene of P22 codes for a repressor, the behavior of which is similar to that of the $\lambda$ repressor (see Levine, 1972, for a review of these studies). Functional $c 2$ gene product is required continuously for the maintenance of lysogeny. However, P22 differs from $\lambda$ in that a second cluster of phage genes is also involved in maintenance of lysogeny and superinfection immunity. Four lines of evidence lead to this conclusion.

$\dagger$ Present address: Pfizer Inc., Groton, Conn. 06340, U.S.A.

$\ddagger$ Present address: Miorobiology and Pharmacology Laboratory, Indian Institute of Science, Bangalore 12, India.

§ Present address: Department of Biology, Stanford University, Stanford, Calif. 94305, U.S.A. 
Zinder (1958) and Gough (1968) studied P22 mutants which define a second gene which must function continuously to maintain lysogeny. This gene, designated mnt, lies a consideruble distance from the $c 2$ gene on the P22 chromosome linked to the $m 3$ plaque morphology marker (Fig. 1). Mutants carrying the mnt-ts allele lysogenize normally at low temperature, but such lysogens are induced at high temperature. The mnt gene must therefore function continuously for the prophage state to be maintained. The $c 2$ and $m n t$ gene products both act in trans, and the wild type alleles of both are dominant to the respective mutant alleles. This implies that they both direct synthesis of diffusible products. These findings suggest that the mnt gene, as well as the $c 2$ gene, specifies a repressor protein.

In crosses between P22 and the related but heteroimmune phage L, Bezdek \& Amati (1968) detected hybrid progeny phages which were still temperate but had immunity characteristics different from each other and from either parent. They reasoned that there must be two immunity regulatory regions in $\mathrm{P} 22$, one linked to the $c$ genes $(\mathrm{imm} \mathrm{C})$ and the other linked to the plaque morphology marker $\mathrm{m} 3$ (imm $\mathrm{I}$ ) (Fig. 1).

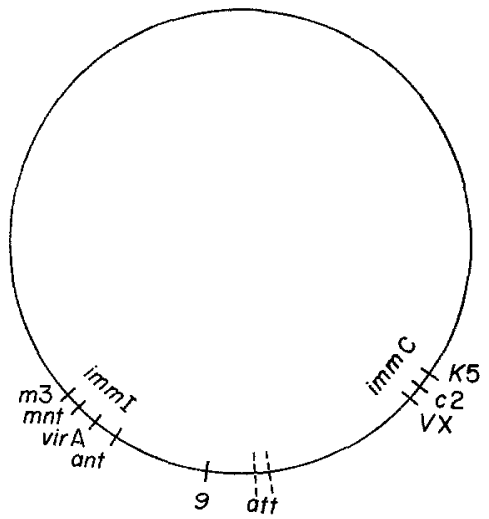

FIG. I. Vegetative map of P22 showing the locations of the $\mathrm{immC}$ and $\mathrm{immI}$ regions.

Both immunity regions of $\mathrm{P} 22$ must be intact to maintain the immunity of a lysogen to superinfection. Defective lysogens which have either the $i m m \mathrm{C}$ or the immI region of the prophage deleted were isolated by Chan \& Botstein (1972) and tested for immunity to homoimmune superinfection. As expected, superinfection of a deletion lysogen lacking the $i m m \mathrm{C}$ region results in normal growth of the superinfecting phage. Deletion lysogens lacking the $\mathrm{immI}$ region are also sensitive to superinfection by homoimmune phage, in spite of the presence of a wild type $c 2$ gene. Thus, both the $i m m \mathrm{C}$ and the $i m m \mathrm{I}$ regions in the prophage must be intact to confer immunity on the lysogenic cell.

Mutations in P22 conferring virulence (the ability of phage to grow in immune lysogens) have been found in both immunity regions. One type, called vir $\mathrm{B}$, consists of two mutations, $V x$ and $K 5$, which lie on either side of the $c 2$ gene (Bronson \& Levine, 1971; Fig. 1). Neither mutation alone causes virulence. The $V \mathbf{x}$ and $K 5$ rnutations appear to be operator mutations which have diminished sensitivity to the c2 repressor (Bronson \& Levine, 1972) analogous to the $v 1, v 3$ and $v 2$ operator mutants 
of phage $\lambda$ (Ptashne, 1971). The other class of virulent mutants, called virA, carries a single mutation which lies very near the $m 3$ locus in the $i m m I$ region (see Fig. 1) (Bronson \& Levine, 1971; Botstein et al., 1975). Mutants of the virA class differ markedly from vir B mutants and from all known $\lambda$ virulent mutants in that they induce wild type prophage on superinfection of a lysogenic host (M. Bronson \& $\mathbf{M}$. Levine, unpublished data; this paper and Botstein et al., 1975). The finding that mutations conferring virulence occur in both the $i m m \mathrm{C}$ and the $i m m \mathrm{I}$ regions again strongly suggests that both regions play important roles in lysogeny.

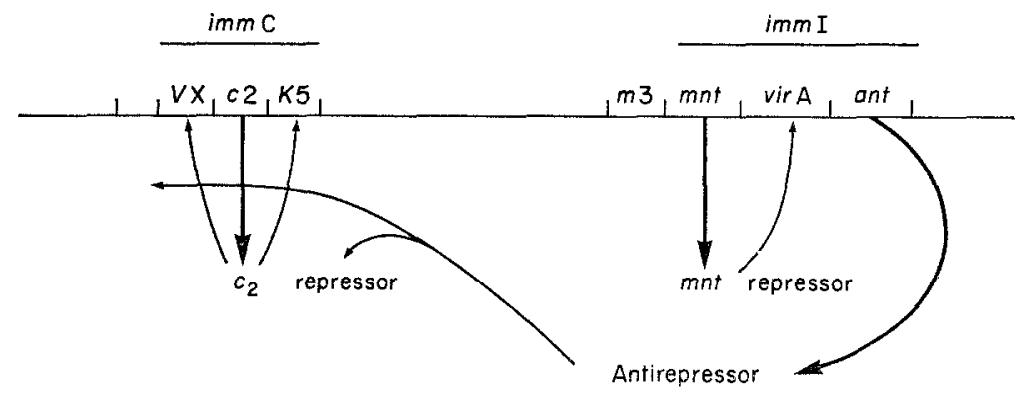

FIc. 2. Proposed interactions within and between the $i m m \mathrm{C}$ and $i m m I$ regions.

On the basis of these findings, Levine (1972) proposed a model for a dual control system of phage P22 which is diagrammed in Figure 2. The model proposes that gene products of the imm C region are responsible for both the establishment and maintenance of repression in lysogeny. The $c 2$ repressor plays the primary role in repression, probably by binding the two operator sites on either side of the $c 2$ gene (the $V \mathbf{x}$ and $K 5$ loci), thereby blocking transcription of genes essential for replication. Levine postulated that the imm I region codes for the synthesis and control of a product which antagonizes the $c 2$ repressor. This hypothetical antirepressor would be specified by a structural gene called ant. The $m n t$ gene product is postulated to be a repressor which regulates the synthesis of the ant gene product. The virA mutants, according to this model, contain mutations in the operator or promoter region governing ant gene expression, making expression of the ant gene no longer sensitive to repression by the $m n t$ gene product. The $m n t$ and virA mutants should therefore produce the antirepressor substance constitutively.

This and the accompanying paper (Botstein et al., 1975) describe the isolation and characterization of antirepressor mutants which have properties predicted by the model. The interaction of the ant locus with the mnt and virA loci provides further support for this model.

\section{Materials and Methods}

(a) Bacterial strains

Salmonella typhimurium strain 18, a derivative of LT2 cured of a defective prophage (Zinder, 1958), was used as the sensitive host for P22. Strain 335 is a strain 18 lysogen obtained from Botstein which carries an $h 21$ int3 sieA6 prophage. It was used as the standard lysogen in this work. Strain DB5057 (Chan \& Botstein, 1972), a tetracycline. resistant, suppressor-negative ( $\left(u^{-}\right)$lysogen lacking the $i m m I$ region of the prophage, was 
also obtained from Botstein, and was used whenever an immI deletion lysogen was required. Strain 2 is a galactose negative mutant derived from strain 18 and was used as indicator on eosin-methylene blue (EMB)-galactose plates. Other lysogens were constructed by infecting strain 18 as described below.

\section{(b) Phage strains}

Strains $c^{+}, c 2^{5}, m n t-t s 1$, and sieAl are from the Levine collection. The mnt mutant was originally isolated by Zinder (1958). The virAl, virA6, virA125 and virB3 mutants were isolated and described by Bronson \& Levine (1971). The ant mutants were isolated as discussed in Results. A mutant carrying an amber mutation in the ant gene, ant-am 19, was obtained from Botstein. Phage strains with combinations of these mutations were constructed by standard crosses (described below). The $c 2$ and $m 3$ phenotypes were used as plaque morphology markers (Levine, 1957) to facilitate identification of recombinants.

\section{(c) Media}

L broth, EMB-galactose agar, green indicator agar and top agar were described by Levine (1957). Tryptone agar (Bronson \& Levine, 1971) was used routinely for plating P22. Strain DB5057, the immI dolotion lysogen, carrios tho baoterial tetraoycline-rosistant allele in the prophage region of its genome. It was grown in $\mathrm{L}$ broth supplement with $25 \mu \mathrm{g}$ tetracycline/ml and plated on similarly supplemented Tryptone agar to stabilize the lysogenic state (Chan \& Botstein, 1972).

\section{(d) Identification of lysogens}

This procedure was deseribed by Levine (1957). On EMB-galactose plates with a lawn of galactose-negative indicator bacteria, galactose-positive strain 18 lysogens appear as dark colonies surrounded by a halo of lysis, while non-lysogens appears as dark colonies with no surrounding halo of lysis. In this way, plaques, lysogens and non-lysogens can be identified on a single plate. EMB plates are incubated at $37^{\circ} \mathrm{C}$ for $24 \mathrm{~h}$ and a further $24 \mathrm{~h}$ at room temperature before scoring.

\section{(e) Segregation experiments}

Segregation experiments to follow establishment of lysogeny were done as described by Smith \& Levine (1967). Strain 18 cells growing exponentially in $\mathbf{L}$ broth were infected with a multiplicity of 20 phage particles/cell at $32^{\circ} \mathrm{C}$. After a 10 -min adsorption period, the cultures were diluted to $10^{3}$ cells $/ \mathrm{ml}$ in broth containing anti-P22 antiserum $(K=3)$. At intervals, samples were plated on EMB-galactose agar as described above for determination of numbers of sensitive and phage-carrying cells.

\section{(f) Infection procedure}

A $1: 100$ dilution of an overnight culture was grown in $L$ broth with aeration at $37^{\circ} \mathrm{C}$ to a concentration of $10^{8}$ cells $/ \mathrm{ml}$. Phage were added at the desired multiplicity and allowed to adsorb for $5 \mathrm{~min}$ at $37^{\circ} \mathrm{C}$. The infected cells were then diluted $1: 10$ into broth containing antiserum (final $K=2$ ) to inactivate unadsorbed phage. Five min later the infected cells were diluted, and a sample plated for infective centers on EMB-galactose plates. The diluted eulture was incubated $90 \mathrm{~min}$ with aration, shakon with a few drops of chloroform, and the progeny phage were assayed with the appropriate indicator bacteria. Superinfection experiments were also carried out in this manner.

\section{(g) Construction of lysogens}

Lysogens were constructed by infecting strain 18 with the appropriate phage at a multiplicity of 10 to 20. Infective centers were plated on EMB-galactose plates as described above. Colonies with haloes of lysis were picked into broth containing anti-P22 antiserum and then streaked for isolated colonies. Colonies were checked for the presence of prophage by testing for immunity by cross-streaking against virulent and non-virulent tester phage. 
Lysogens to be used as hosts for superinfection carry a sieA mutation in the prophage. The sie mutation prevents exclusion of superinfecting phage (Walsh \& Meynell, 1967; Rao, 1968; Susskind et al., 1971).

\section{(h) Mutagenesis}

Strain 18 cells growing exponentially in $\mathrm{L}$ broth at $37^{\circ} \mathrm{C}$ were infected with the appropriate phage at multiplicities of 5 to 10 in the presence of $4 \mu \mathrm{g} 1$-methyl-3-nitro-l-nitrosoguanidine $/ \mathrm{ml}$. The infected cultures were incubated long enough to allow one cycle of phage growth, and the progeny were plated with sensitive strain 18 cells as indicator.

TABLE 1

Identification of phage mutants

\begin{tabular}{lcccc}
\hline Phage type & $\begin{array}{c}\text { Growth on } \\
\text { a lysogen }\end{array}$ & $\begin{array}{c}\text { Bullseye } \\
\text { plaque } \\
\text { morphology } \dagger\end{array}$ & $\begin{array}{c}\text { Colony } \\
\text { appearance }\end{array}$ & $\begin{array}{c}\text { Growth on } \\
\text { imm I deletion }\end{array}$ \\
\hline Wild type & - & - & Pale green & + \\
mnt & - & + & Dark green & + \\
virA & + & + & Dark green & + \\
ant & - & - & Pale green & - \\
\hline
\end{tabular}

$\dagger$ The bullseye morphology is a plaque with a slightly turbid center containing a series of concentric rings of bacterial growth.

† The eolor of bacteria picked from plaque centers, stabbed unto green indicator agar and incubated at $25^{\circ} \mathrm{C}$ for 36 to $48 \mathrm{~h}$.

\section{(i) Identification of mutants in the immI region}

Wild type, mnt, virA, and ant phage can be distinguished by growth on various host strains arnd by plaque morphology, as shown in Tuble 1. Only virA mutants grow on the standard lysogen. Both virA and mnt phage have a distinctive "bullseye" plaque morphology (the plaques are slightly turbid with a series of concentric rings of bacterial growth) and fail to lysogenize stably. Colonies picked for the slightly turbid centors of virA or mnt plaques give a dark green color on green indicator plates without a lawn of bacteria. Stable lysogenic colonies are pale green on such plates (Smith \& Levine, 1967). All ant ${ }^{+}$phages are able to grow on immI deletion lysogens (Chan \& Botstein, 1972). As will be shown in this report and the accompanying one (Botstein et al., 1975), ant phage cannot grow on $i m m I$ deletion lysogens. Thus the presence of these markers can be determined by picking plaques with sterile toothpicks and stabbing into Tryptone plates with a sensitive lawn, Tryptone plates with a lysogenic lawn (strain 335), green plates without a lawn, and Tryptone + tetracycline plates with the immI deletion lysogen lawn. In addition, the plaque morphology marker $m 3$ can be identified by the characteristic yellow. ish halo it gives to the plaques on green indicator plates with a strain 18 lawn (Levine, 1957).

\section{Results}

\section{(a) Comparison of the two types of virulent mutants}

Virulent mutants can grow in lysogenic cells in which the growth of superinfecting non-virulent phage is repressed (Table 2). In lysogenic cells, at multiplicities of five to ten, $\mathrm{P} 22$ phages of both vir $\mathrm{A}$ and vir $\mathrm{B}$ classes yield more than 100 particles per infected cell, whereas non-virulent $c 2$ mutants produce fewer than one progeny particle per infected cell. However, the population of progeny phages varies in the two types of virulent superinfections. Superinfection of a lysogen with a virB mutant yields 


\section{TABLE 2}

Ability of $\operatorname{vir} A$ and $\operatorname{vir} B$ mutant phage to permit prophage or co-infecting phage growth after infection of a lysogen

\begin{tabular}{|c|c|c|c|}
\hline $\begin{array}{c}\text { Superinfecting } \\
\text { phage }\end{array}$ & Burst size & \multicolumn{2}{|c|}{$\begin{array}{l}\text { Ratio of phage } \\
\text { types in yield }\end{array}$} \\
\hline $\operatorname{vir} \mathrm{Al}$ & 130 & $\operatorname{virA}: c^{+}$ & $2: 1$ \\
\hline$c 2$ & $0 \cdot 2$ & $c 2: c^{+}$ & $15: 1$ \\
\hline $\operatorname{vir} \mathrm{A} 1+c 2$ & 210 & $\operatorname{vir} \mathrm{A}: c 2: c^{+}$ & $4: 2: 1$ \\
\hline $\operatorname{virB3}$ & 410 & $\operatorname{vir} \mathrm{B}: c^{+}$ & $50: 1$ \\
\hline $\operatorname{vir} \mathbf{B}+c 2$ & 110 & $\operatorname{vir} \mathrm{B}: c 2: c^{+}$ & $50: 1: 1$ \\
\hline
\end{tabular}

Infections were done in 18 (sie 6 int 321 ) in $L$ broth at an m.o.i. of 5 to 10 each. Burst size is defined as the number of progeny/number of cells exposed to phage. Each infecting phage has a distinctive morphology which permits it to be distinguished from the prophage under our standard plating conditions.

almost exclusively vir $\mathrm{B}$ progeny, because vir $\mathrm{B}$ phage cannot overcome the repression of either a co-infecting non-virulent phage or the prophage (Table 2 ; Bronson \& Levine, 1971,1972 ). The inability of $\operatorname{vir} B$ mutants to relieve the repression of non-virulent P22 genomes is due to replication inhibition of the non-virulent genomes by the $c 2$ gene product, as association of the non-virulent DNA with the phage replicative complex is blocked (Levine et al., 1970). In contrast, superinfection of a lysogen with a virA mutant yields not only virulent progeny, but also a high proportion of prophage and co-infecting non-virulent progeny types (Table 2). This finding indicates that virA mutants overcome the repression of non-virulent phage in the same cell. The ability of virA mutants to relieve the repression of non-virulent phage in a lysogen is interpreted according to the hypothesis given in the Introduction as evidence for the constitutive synthesis by the virA phage genome of an anti-crepressor which acts in trans. A search for the locus encoding such a substance was undertaken.

The accompanying paper by Botstein et al. gives a more extensive characterization of virA type mutants. It should be pointed out that they isolated their virA mutants by selecting for spontaneous mutations causing instability of lysogeny and then screened for the ability to kill lysogens. The virA mutants isolated by Bronson \& Levine (1971) and used by us in this report were selected directly for plaque formation on lysogens. The mutants show a fully virulent phenotype, giving high yields of progeny phage in lysogenic cells. In contrast, the analogous mutants of Botstein et al. (1975), which they call $V_{y}$, vary in phenotype. Some are only able to make plaques on lysogens when combined with $c 2$ mutation. Others do make plaques on lysogens, but need a $c 2$ mutation to produce a substantial yield of progeny. We have agreed that only fully virulent phage strains are to be called virA. In Botstein et al., 1975, the combination $V y c 2$ is called $v i r A$, while our single mutant full virulents are called virA.

\section{(b) Isolation and characterization of $\operatorname{vir} A$ ant double mutants}

If the virulence of virA mutants is due to constitutive synthesis of antirepressor, a defect in the structural gene for the antirepressor should cause loss of virulence. 
Three independently isolated virA mutants, virAl, virA6, and virAl25, were mutagenized with nitrosoguanidine. Single plaques were picked onto replicate plates, seeded with sensitive or lysogenic bacteria, to screen for mutants which had lost the ability to grow on the lysogen while retaining the ability to plate on the sensitive host. Several such isolates from each virulent strain were purified from the sensitive host plate and studied further.

To show that these non-virulent mutants still carried the original virA mutation, test crosses were carried out between isolates from each virA strain and wild type phage. In all cases, virulent progeny were detected at low but significant frequencies. Therefore these non-virulent isolates are not simply revertants of the original virA mutation, but rather contain an additional mutation(s) which is (are) responsible for the loss of virulence.

Evidence that all the non-virulent isolates are deficient in the same function comes from complementation tests. If the different non-virulent derivatives of virA carry mutations in different genes, they should be able to complement each other and restore virulence on mixed superinfection of a lysogen. Equivalent burst sizes in sensitive and lysogenic hosts would be taken as evidence for complementation. The non-virulent isolates derived from each virA strain were tested with the other isolates from the same strain. None of these pairwise combinations of non-virulent mutants produced more than one progeny particle per cell in a lysogenic host. Then a representative non-virulent isolate from each of the three virA parents was tested against representatives of the other groups. None of these mutants complemented any of the others, because all mixed infections in the lysogenic host gave yields of less than one progeny particle per infected cell. Mixed infections of the non-virulent isolates in the sensitive host produced more than 100 progeny particles per cell, and the parental virA phage produced more than 100 particles per cell in both hosts. These results suggest that all the newly induced second mutations are in the same cistron.

These second mutations show two properties predicted for the postulated ant mutations. Phage carrying these mutations show loss of vir A virulence and inability to grow in immI deletion lysogens (Table 5, line 2; Table 6; Botstein et al., 1975). According to the model, immI deletion lysogens are sensitive to superinfection by homoimmune phage because the deleted prophage cannot synthesize the mnt repressor which normally represses expression of the ant gene of the superinfecting phage. However, if the superinfecting phage lacks a functional ant gene, it should not grow in an immI deletion lysogen. The finding that the non-virulent derivatives of virA mutants do not grow in immI deletion lysogens makes it unlikely that they contain a second mutation within the virA locus, because all known virA mutants grow in such lysogens. Therefore, the non-virulent phage derived from virA are assumed to be virA ant double mutants.

The double mutant virA ant phage exhibit none of the characteristics of the parental vir A phage. On superinfection of a lysogen, virA ant phage not only produce very few progeny, but also do not relieve the repression of prophage and co-infecting nonvirulent phage (Table 5, lines 2 and 6 ). Their plaque morphology more closely resembles that of wild type phage than that of the parental virA phage. They do not grow on immI deletion lysogens as do virA mutants. In contrast to virA phage, virA ant phage are able to lysogenize normally as described below.

Mutants of the virA type are unable to form stable lysogens as judged by the dark green color of cells picked from the centers of the slightly turbid plaques onto green 

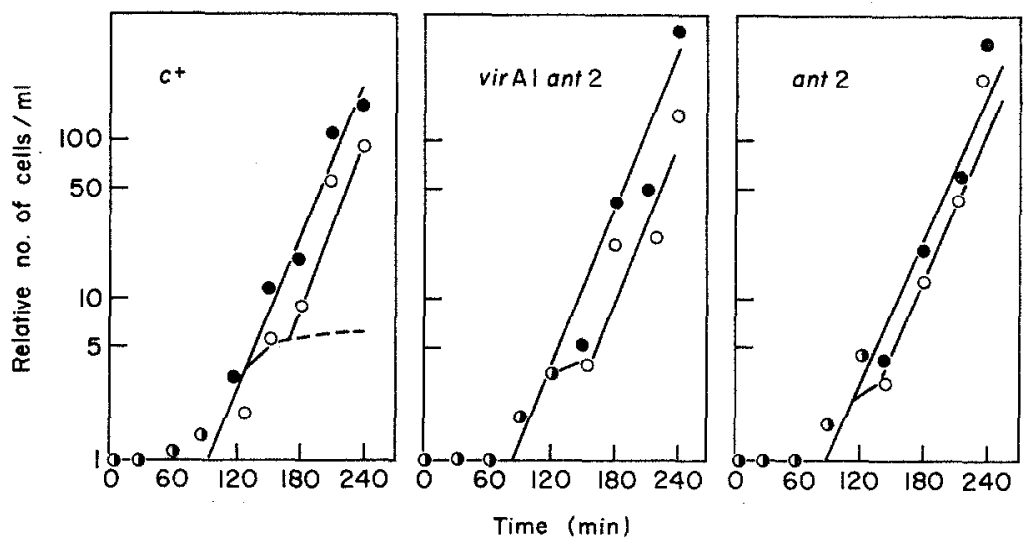

Fia. 3. Segregation of phage carrying and sensitive cells following infection by $c^{+}$, virAl ant $2 c^{+}$ or ant $2 c^{+}$phage. The experiments were done at $32^{\circ} \mathrm{C}$ using a multiplicity of 20 phage particles. Cell numbers are relative to the initial number of infected cells. The dashed line in the $c^{+}$figure represents the relative number of phage carriers in an int infection for comparison (see text). (C) Total cells; (O) phage-carrying cells.

indicator agar. Cells from $c^{+}$plaques show a pale green color on green indicator plates. Cells from the virA ant mutant plaques also show pale green growth on the indicator plates. These double mutants are able to lysogenize sensitive bacteria, and at frequencies equivalent to that of wild type phage. This was demonstrated by a study of the segregation patterns of infected cells as described in Materials and Methods. Smith \& Levine (1967) showed that following infection of a sensitive host by $c^{+}$ phage, the proportion of phage-carrying cells to total cells remains high for a few generations, but eventually cells segregate as sensitives or stable lysogens (Fig. 3). Prophage integration is assumed to occur during the segregation period, because thereafter the capacity to produce phage is perpetuated in parallel with cell growth. A very different segregation pattern is observed for P22 mutants, such as int mutants (Smith \& Levine, 1967), which cannot accomplish a step necessary for prophage integration. In this case, a high proportion of cells initially survives as phage carriers, but after some increase in cell titer, the ratio of phage carriers to total cells decreases with each division, indicating the absence of stable lysogeny. (The dotted line in Fig. 3 indicates this effect.) Infection of strain 18 cells with virAl ant $2 c^{+}$mutants gives a similar segregation pattern to that of $c^{+}$phage (Fig. 3). That is, virA ant mutant phage can lysogenize just as efficiently as wild type P22. This result demonstrates that the inability of virA mutants to lysogenize is directly related to the functioning of the ant gene.

Attempts to find mutants starting with $\operatorname{vir} \mathrm{B}$ phage which have lost virulence and in this sense are analogous to virA ant mutants were unsuccessful.

\section{(c) Isolation and characterization of mnt-ts ant double mutants}

The model predicts that absence of $m n t$ product results in constitutive synthesis of antirepressor just as a virA mutation does. Thus it should be possible to isolate mnt-ts ant double mutants which do no longer exhibit the mnt-ts phenotype. A search for such mutants was undertaken. As described in Materials and Methods, mnt-ts 
phage makes plaques at the non-permissive temperature that are clearer than wild type and have a "bullseye" morphology. The mnt-ts mutants form stable lysogens at low temperature, but cannot maintain the prophage state at high temperature (Gough, 1968). If the model is correct, mnt-ts ant double mutants should make plaques which are turbid rather than bullseye, and lysogens carrying a mnt-ts ant prophage should not be inducible at high temperature.

A stock of P22 mnt-ts phage was mutagenized with nitrosoguanidine and plated at high temperature on strain 18. Phage from 12 turbid plaques were isolated and purified for further study. Cells from the centers of these plaques, picked onto green indicator agar plates, give pale green growth at high temperature which is indicative of stable lysogeny. This is to be contrasted with the dark green growth of cells from plaques of the original mnt-ts phage.

These isolates were test-crossed with wild type phage to demonstrate that they still contain the mnt-ts mutation. Recombinants were found among progeny of these crosses which, at high temperature, show bullseye plaque morphology and give dark green colony formation when these plaques are pjcked onto green indicator plates. Therefore these isolates are not simply revertants of the mnt-ts mutation but contain an additional mutation(s) which is (are) responsible for the loss of the mnt-ts phenotype.

These double mutant phage, which no longer express the mnt-ts phenotype, do not grow on $\mathrm{immI}$ deletion lysogens as expected of ant mutants. Mixed infections between these isolates in the immI deletion lysogen did not produce phage. The yields were less

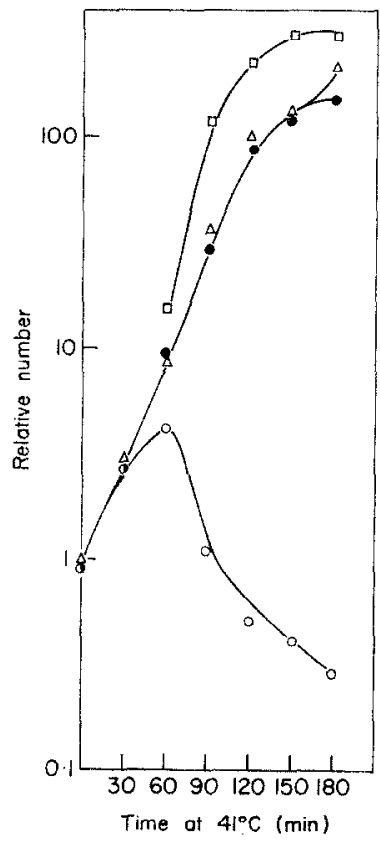

FIG. 4. Thermal induction of $18\left(c^{+}\right), 18\left(m n t-t s 1 c^{+}\right)$and $18\left(m n t-t s 1\right.$ ant5 $\left.c^{+}\right)$lysogens. Cells were grown in $\mathrm{LB}$ broth at $25^{\circ} \mathrm{C}$ to a concentration of $5 \times 10^{7}$ cells $/ \mathrm{ml}$ and then shifted to $41^{\circ} \mathrm{C}$. Samples were taken at the indicated times and plated at low temperature for colony formation and for phage production.

(D) Phage produced by $18\left(m n t-t s 1 c^{+}\right) ;(\triangle)$ 18(mnt-tsl ant5 $\left.c^{+}\right)$colony formers; $(9)$ 14 $\left(c^{+}\right)$ colony formers; $(O) 18\left(m n t-t s 1 \mathrm{c}^{+}\right)$colony formera: 
than one progeny particle per superinfected cell compared to yields of more than 100 in sensitive cells. These data strongly suggest that the second mutations induced in these strains all lie in the same cistron, and that the isolates are mnt-ts ant double mutants.

These double mutant phage can form stable lysogens which are no longer inducible at high temperature. Figure 4 shows the comparative inducibility at $41^{\circ} \mathrm{C}$ of $18\left(c^{+}\right)$, $18\left(m n t-t s 1 c^{+}\right)$and $18\left(m n t-t s 1\right.$ ant5 $\left.c^{+}\right)$lysogens. The cells were grown in $\mathrm{L}$ broth at $25^{\circ} \mathrm{C}$ to a titer of $5 \times 10^{7}$ cells $/ \mathrm{ml}$ and then shifted to $41^{\circ} \mathrm{C}$. At intervals, samples were plated at low temperature for colony formation and for phage production. As previously described (Gough, 1968), the 18(mnt-tsl $c^{+}$) culture undergoes a few divisions and then begins to lyse giving a burst of progeny phage. In contrast, the $18\left(\mathrm{c}^{+}\right)$and 18(mnt-ts 1 ant $\left.5 c^{+}\right)$lysogenic cultures show parallel increases in cell number with no evidence of cell lysis and phage production. This result shows that the ant function is necessary for thermal induction of mnt-ts mutant prophage.

The mnt-ts ant lysogens are, however, still inducible by ultraviolet light. Inducing doses of ultraviolet light cause induction of comparable numbers of wild type and mnt-ts ant lysogens to produce comparable yields of progeny phage. The ant gene function, therefore, is not required for induction by ultraviolet light (see also Botstein et al., 1975).

\section{TABLE 3}

Mapping of $\operatorname{vir} A$, mnt-ts and ant

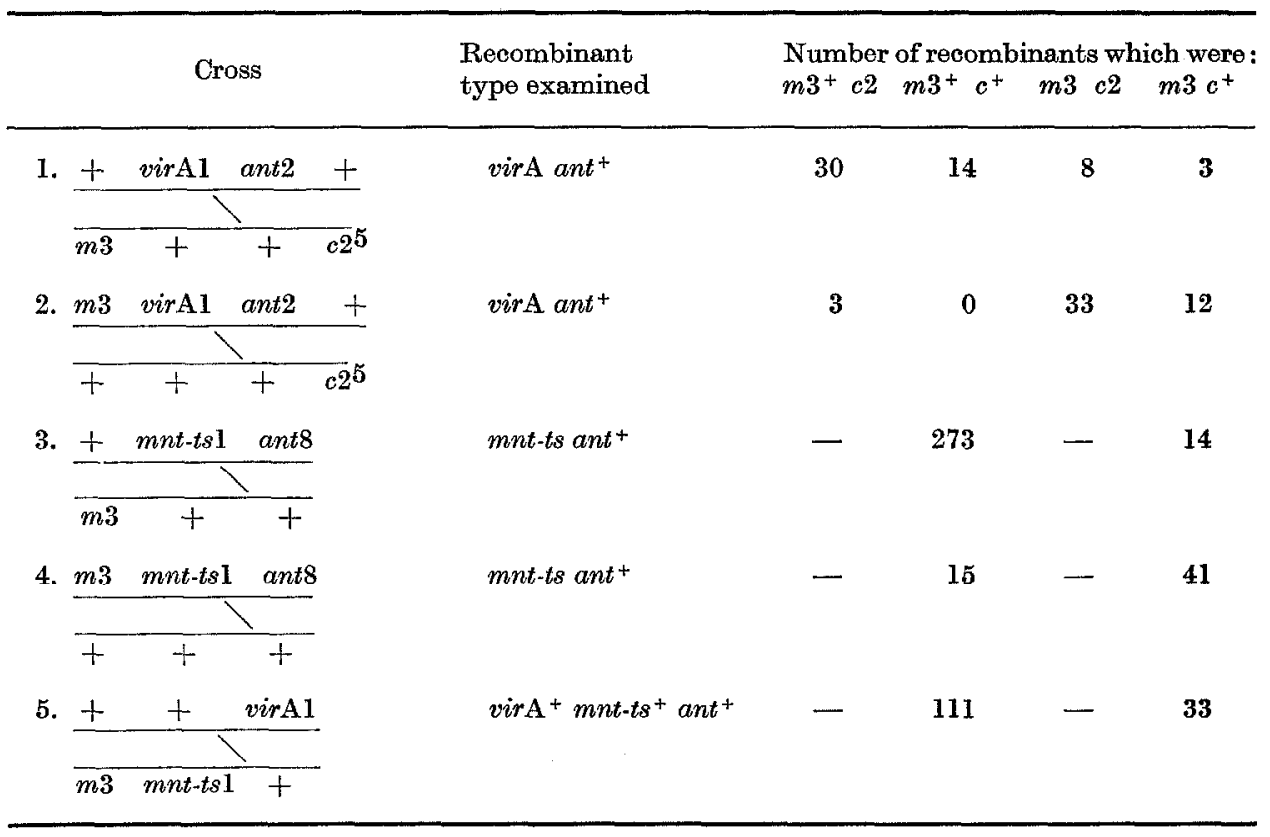

Crosses were done in the standard manner described in Materials and Methods using a multiplicity of 5 of each parent. The progeny were analyzed by picking from Tryptone agar plates onto various backgrounds as shown in Table 1 and described in Materials and Methods. The virA $c 2$ recombinants were classified as phage which grow on the standard lysogen and also produce clear plaques on the standard sensitive strain (that is they do not show the characteristic bullseye morphology of virA $c^{+}$phage). 
(d) Mapping of mnt, $\operatorname{vir} A$ and ant mutants

Gough (1968) mapped the $m n t$ locus between the $m 3$ and gene 9 loci. Bronson \& Levine (1971) presented data that the virA locus is closely linked to $m 3$. Evidence is given below showing that the ant locus lies in this region, and that the order of loci is m3-mnt-virA-rnt--co2 (Fig. 1).

The virA and ant mutations were oriented relative to each other and to $m 3$ and $c 2$ in crosses 1 and 2 shown in Table 3. Virulent recombinants were detected, and each was classified as to plaque morphology as described in Materials and Methods. Reasoning that the least frequent recombinant class requires the most crossovers and the most frequent class the fewest, the order $m 3$-virA-ant-c2 was deduced.

The order of the loci $m n t-t s$, ant and $m 3$ was determined by three-factor crosses (lines 3 and 4 in Table 3 ). The $m n t$-ts recombinants were detected and each classified as to its $m 3$ morphology. The data suggest the order $m 3-m n t-a n t$.

Finally, the mnt-ts and virAl mutations were ordered relative to each other and to the $m 3$ locus in the cross virAl $\times m 3 m n t$-ts (line 5, Table 3). In this case, recombinants among the progeny which had wild type alleles at the virA and $m n t$ loci were detected by the more turbid appearance of the plaques, and classified as to $m 3$ morphology. The order of these loci is m3-mnt-virA.

The order of all the loci studied is m3-mnt-virA-ant-c2. This order is in agreement with that determined by Botstein et al. reported in the accompanying paper. The four loci $m 3$, mint, virA and $u n l$ are very closely linked. Although no attempt was made to obtain precise linkage data, all these loci lie within two linkage units of one another.

\section{(e) Isolation of ant mutants by recombination and their characterization}

To study the effects of a mutation in the ant gene on the properties of otherwise wild type phage, strains carrying only an ant mutation were constructed. The double mutants virAl ant2 and mnt-ts ant8 were each crossed with ant ${ }^{+}$phage carrying the outside markers $m 3$ and $c 2$ (see Table 3 , lines 1 and 3). Phage carrying ant mutations were identified among progeny recombinant for the outside markers by their failure to grow on the immI deletion lysogen. Plaques of a number of ant phage were purified and test-crossed with wild type phage in order to distinguish ant from virA ant or $m n t$-ts ant. The progeny of an ant $\times$ wild-type cross should include nor virA or mnt-ts phage. In this manner, an $m 3$ ant 2 strain and an $m 3$ ant8 strain were obtained from virAl ant2 and mnt-ts1 ant8, respectively.

These ant mutants grow on sensitive bacterial strains, which demonstrates that the ant gene product is not essential for lytic growth (see Table 6). They form turbid plaques on strain 18 which are indistinguishable from wild type plaques. Phage P22 ant mutants are not virulent. Segregation experiments with the ant 2 mutant showed that it is capable of lysogenizing normally (Fig. 3), demonstrating that the ant gene product is not necessary for the establishment or maintenance of lysogeny. The ant mutants do not grow in immI deletion lysogens in contrast to other P22 phage which grow well in such lysogens (Table 4). Botstein et al. (accompanying paper) have isolated ant mutants by selection for inability to grow in immI deletion lysogens. Their ant mutants do not complement ours (see next section) and behave identically. Because the characteristics of ant mutants are quite different from those of virA or mit mutants, it is reasonable to conclude that ant mutants are at a different locus from either virA or mnt mutations. 
Table 4

Complementation tests showing that all ant mutations are in the same cistron

\begin{tabular}{lc}
\hline Phage & Burst size \\
\hline 1. Wild type & 370 \\
2. $m 3$ ant 2 & $0 \cdot 6$ \\
3. $m 3$ ant8 & $0 \cdot 1$ \\
4. ant-am $19 \dagger$ & $0 \cdot 3$ \\
5. $m 3$ ant $2 \times$ wild type & $140 \ddagger$ \\
6. $m 3$ ant $8 \times$ wild type & $100 \ddagger$ \\
7. ant-am $19 \times$ wild type & 355 \\
8. $m 3$ ant $2 \times m 3$ ant 8 & $0 \cdot 1$ \\
9. $m 3$ ant $2 \times$ ant-am 19 & $0 \cdot 2$ \\
10. $m 3$ ant $8 \times$ ant-am 19 & $0 \cdot 2$ \\
\hline
\end{tabular}

These infections were carried out in the $s u^{-} i m m I$ deletion lysogen at an m.o.i. of 5 each. The progeny were assayed with a non-lysogenic indicator strain.

$\dagger$ This mutant carries an amber mutation in the ant gene. It was obtained from Botstein.

$\ddagger$ Both $m 3$ and $m 3^{+}$plaque morphology markers were found among the progeny phage in approximately equal numbers.

To determine whether all the ant mutations are in the same cistron complementation tests between our ant2 and ant8 mutants and the Botstein's group's ant-am19 mutant were performed in the $s u^{-} \mathrm{immI}$ deletion strain (Table 4). As already described, ant ${ }^{+}$phage grow in these lysogens, but ant mutants do not. All three of the ant mutants tested can be complemented by wild type phage as judged by the appearance of approximately equal numbers of each parental type progeny in the yields. Very few phage are produced in pairwise mixed infections by the three ant mutants. These data support the conclusion that all the ant mutations are in the same cistron, whether isolated in a virA or mnt-ts background or by inability to plate on un immI deletion lysogen. The data also show that the ant locus acts in trans, because the ant function performed by the wild type phage permits the growth of the coinfecting ant mutant phage as well.

Additional evidence that the ant mutations from different sources are equivalent comes from experiments with virAl ant8 and mnt-tsl ant2 douboe mutant strains which were constructed by recombination. The ant 8 mutation, originally derived from a mnt-ts ant8 double mutant, was crossed into a virAl mutant phage. The virAl ant8 double mutant has properties indistinguishable from those of virAl ant mutants isolated by mutagenesis of virAl. It is no longer virulent, can lysogenize, and cannot plate on an immI deletion lysogen. In the reciprocal experiment, the ant 2 mutation which was originally isolated as a virAl ant2 double mutant was crossed with a $m n t$-ts phage. The mnt-ts ant 2 double mutant phage has the same properties as the mutants isolated by mutagenesis of mnt-ts. These results support the conclusion that the ant mutations, regardless of the method of their isolation, are in the same gene.

(f) Mutations in the virA locus uffect only the ant gene on the same chromosome

The model predicts that virA mutations are lesions in the operator or promoter region which controls synthesis of the ant gene product. If this is so, virA mutations 
TABLE 5

Complementation tests showing that vir $A$ mutations affect only the ant gene on the same chromosome

\begin{tabular}{lc}
\hline \multicolumn{1}{c}{ Phage } & Burst size \\
\hline 1. virAl ant $^{+}$ & 125 \\
2. virAl ant2 & $0 \cdot 25$ \\
3. ant & $0 \cdot 04$ \\
4. ant2 & $0 \cdot 05$ \\
5. virAl ant $^{+}+a n t^{+}$ & $110 \dagger$ \\
6. virAl ant $^{+}+a n t^{+}$ & $0 \cdot 17$ \\
7. virAl ant + ant2 & $50 \dagger$ \\
8. virAl ant2 + ant2 & $0 \cdot 23$ \\
\hline
\end{tabular}

Infections were done in our standard lysogen $18(\operatorname{sie} 6 \mathrm{int3} \mathrm{h2l})$ at multiplicities of 6 each. Progeny phage were assayed with a non-lysogenic indicator strain. The burst size is defined as in Table 2.

$\dagger$ Both $c^{+}$and virA progeny were present in the yield.

should affect the expression only of the ant gene on the same chromosome. This postulate of the model is supported by the result of a cis-trans complementation test in a lysogenic host. Various combinations of virA and ant mutations and their wild type alleles were tested for complementation for growth (Table 5). Lines 6 and 7 show the results of the crucial experiments. In the infection shown on line 6 the virA mutation was in trans to the wild type ant gene, while in the infention on line 7 the $\operatorname{vir} \mathrm{A}$ mutation was in cis to the wild type ant gene. Only when virA and the wild type ant gene are in a cis configuration are progeny phage produced. Therefore it can be concluded that virA mutations affect only the ant gene on the same chromosome. This experiment also provides further evidence that the ant locus acts in trans, because in the infection shown on line 7, the ant function provided by the virA ant ${ }^{+}$ phage permits the growth of the ant phage as well.

(g) The mnt repressor is needed for immunity to superinfection

Lysogens with the $\mathrm{immI}$ region of the prophage deleted are sensitive to superinfection by homoimmune phage (Chan \& Botstein, 1972). It was of interest to determine which gene or genes in this region is or are responsible for immunity to superinfection. This was done by examining the response to superinfection of lysogens carrying prophage with different combinations of $i m m I$ mutations. The lysogens carried one of five types of prophage: wild type with respect to the $i m m I$ region $\left(i m m I^{+}\right)$, an immI deletion lacking the entire $i m m I$ region, ant2, virAl ant2, or mnt ant8. Infections of the lysogens were carried out following the procedure described in Materials and Methods. Typical results are shown in Table 6.

As expected, non-virulent phage cannot grow in an $i m m \mathrm{I}^{+}$lysogen. In a lysogen whose prophage lacks the $i m m I$ region completely, any superinfecting phage which has a functional ant gene can grow, as originally observed by Chan \& Botstein (1972) and Botstein et al. (1975). A prophage with a mutation in the ant gene prevents growth 
TABLE 6

Superinfection of lysogens containing mutations in the immI region

\begin{tabular}{|c|c|c|c|c|c|c|c|}
\hline Phage/Bacteria & $c^{+}$ & ant $2 c^{+}$ & $m n t$ & $c 2$ & ant $2 \mathrm{c2}$ & virA & $\operatorname{virA} a n t 8$ \\
\hline 18 & 320 & 50 & 400 & 440 & 40 & 440 & 250 \\
\hline $18\left(i m m \mathrm{I}^{+}\right)$ & $0 \cdot 1$ & $0 \cdot 1$ & $0 \cdot 1$ & $0 \cdot 1$ & 0.06 & 100 & $0 \cdot 5$ \\
\hline $18(\mathrm{immI}$ deletion $)$ & 125 & 0.05 & 140 & 205 & 0.5 & 250 & $0 \cdot 6$ \\
\hline $18(\operatorname{ant} 8)$ & $0 \cdot 1$ & $0 \cdot 05$ & $0 \cdot 4$ & $0 \cdot 4$ & $0 \cdot 3$ & 66 & 0.3 \\
\hline 18(virA1 ant8) & 0.5 & $0 \cdot 4$ & 1.0 & 0.4 & 0.2 & 81 & $0 \cdot 3$ \\
\hline $18(m n t$ ant 8$)$ & 36 & $0-3$ & 34 & 235 & $0 \cdot 0$ & 45 & $0 \cdot 6$ \\
\hline
\end{tabular}

The numbers are the burst sizes defined as in Table 2. The multiplicity of infection was 5 to 10.

of non-virulent superinfecting phage just as well as a wild type prophage does. Thus the product of the ant gene plays no role in protecting a lysogen against superinfection. A virAl ant8 lysogen also behaves much like an $i m m I^{+}$lysogen. This result demonstrates that the sensitivity of the immI deletion lysogen is not due to lack of virA or ant gene functions. Only the mnt ant8 lysogen is sensitive to superinfection by the same phages which grow on the $i m m I$ deletion lysogen; that is, all ant ${ }^{+}$phages replicate, while ant mutants do not. It can be concluded from this similarity that it is the lack of $m n t$ repressor in both the $i m m I$ deletion and mnt ant8 lysogens which makes them sensitive to superinfection by ant ${ }^{+}$phage. The observation that mnt ant 8 lysogens are sensitive to superinfection by ant ${ }^{+}$but not by ant phage further supports the contention that the mnt gene product functions by preventing synthesis of the ant gene product. It should be noted that the mnt ant lysogen has not completely lost superinfection immunity because it is still immune to superinfection by ant mutant phage.

\section{Discussion}

\section{(a) Properties of the ant locus}

The model described in the Introduction (Fig. 2; Levine, 1972) predicts the presence of an antirepressor locus in the immT region of the phage P22 genome. Mutants at this locus were isolated as second mutations in virA and $m n t-t s$ phages which result in the loss of the virA and mnt-ts mutant phenotypes. These ant mutations were separated from the virA and $m n t$ loci by recombination and have the following properties. The ant mutants grow well in sensitive cells, demonstrating that the ant function is not essential for phage production. They make turbid plaques on sensitive cells indistinguishable from wild type plaques. The ant mutants are able to lysogenize normally, and ant lysogens are immune to superinfection by homoimmune phage. Thus, ant function is not required for establishment or maintenance of the prophage state or for the expression of superinfection immunity. The ant mutant lysogens are inducible both by ultraviolet irradiation and by high temperature if the prophage carries a $c 2$-ts allele (S. Truesdell \& M. Levine, unpublished results; Botstein et al., 1975). This demonstrates that ant gene activity is not required for induction of phage production, nor is it directly involved in repression of phage $\mathbf{P} 22$ replication by the gene $c 2$ repressor. In contrast to $a n t^{+}$phage, ant mutants superinfecting $i m m I$ deletion lysogens or $m n t$ 
ant lysogens cannot produce progeny. The normal activity of the ant locus is required for growth of superinfecting phage in these cells. Whether isolated originally as virA ant or mnt-ts ant double mutants, ant mutations do not complement for phage production in immI deletion lysogens. Thus virA and $m n t$ phages require the activity of the same locus, the ant locus, for expression of their respective phenotypes. The ant gene acts in trans, suggesting that it is the structural gene for a diffusable product. The isolation of temperature-sensitive and amber mutations at this locus (Botstein et $a l ., 1975$ ) provides evidence that this product is a protein.

\section{(b) The mnt and $\operatorname{vir} A$ loci control ant gene expression}

The model (Levine, 1972) suggests that the mnt and virA loci regulate the ant locus, the $m n t$ locus coding for a repressor, and the virA locus acting as an operator or promoter for ant gene expression. The data presented in this and the accompanying paper (Botstein et al., 1975) are consistent with this suggestion.

Gough (1968) has shown that the product of the mnt gene is required continuously for maintenance of stable lysogeny, that the $m n t$ gene product acts in trans, and that the $m n t^{+}$allele is dominant to $m n t$. He interpreted these facts to mean that the $m n t$ gene product acts as a repressor. Additional cvidence in support of this interpretation comes from the superinfection experiments reported in this paper. Lysogens carrying mnt mutant prophage, necessarily mnt ant double mutant prophage, are no longer immune to superinfection by $a n t^{+}$phage. Lysogens carrying ant or virA ant mutant prophages retain immunity to superinfection. Thus, the active expression of the $m n t$ gene in the $\mathrm{immI}$ region is needed for repression of the growth of $a n t^{+}$superinfecting phage. It can be concluded that it is the absence of the $m n t$ locus in the $i m m$ I deletion lysogens which makes them susceptible to superinfection.

The mnt gene product acts by controlling the ant gene product. This is shown by two lines of evidence. First, mnt ant double mutant phage no longer exhibit the mnt phenotype. In contrast to single mnt mutants they lysogenize as stable prophages, and mnt-ts ant prophages are not inducible at high temperature. That is, the expression of the mnt mutant phenotype requires a functional ant locus. Second, the finding that the mnt ant lysogens are sensitive to superinfection by ant ${ }^{+}$but not by ant phages suggests that the $m n t$ gene product plays its role in superinfection immunity by repressing the synthesis of the ant gene product.

Virulent phages of the virA type are inducing phages. Not only do they grow in lysogens, but they also induce prophage and overcome replication inhibition of coinfecting nonvirulent phage (Levine et al., 1970). The virA phenotype depends on the presence of a functional ant locus on the virA mutant genome. Double mutants of the virA ant type are not virulent and do not show the other properties of virA mutants. Further, no progeny phages are produced in mixed superinfection of a lysogen by virA ant and wild type phage indicating that the virA locus is cis dominant, affecting only the expression of the ant gene on the same chromosome. Finally, the virA and ant loci are adjacent to each other on the P22 linkage map. These findings are consistent with the idea that the virA locus is either the operator or promoter region controlling expression of the ant gene. Mutations in the virA locus result in constitutive synthesis of the ant gene product.

As might be expected of loci which both control the expression of the same gene, virA and mnt mutants are similar in several respects. Both types of mutants are 
incapable of forming stable lysogens and give rise to plaques with a slightly turbid bullseye morphology. These phenotypic similarities are undoubtedly dependent on the constitutive synthesis of the ant gene product by both mutants.

\section{(c) The function of the ant gene product}

The evidence presented in this and the accompanying paper (Botstein et al., 1975) is consistent with the model proposed by Levine (1972) for the gene structure and interactions of the loci of the $i m m \mathrm{I}$ region. Based primarily on the inducing action of virA mutants, the model also suggests that the action of the ant gene product is to antagonize the gene $c 2$ repressor, the primary repressor of phage $\mathrm{P} 22$ replication. The antirepressor action of the ant gene product on the $c 2$ repressor can be visualized in a number of ways.

The ant gene product might act by preventing synthesis of the $c 2$ repressor in a manner analogous to the action of the phage $\lambda$ gene cro on the $\lambda c I$ repressor (Reichardt \& Kaiser, 1971 ; Echols et al., 1973). The action of the cro locus is not well understood but is thought to prevent transcription of some of the early classes of $\lambda$ messenger RNA (Court \& Campbell, 1972; Echols, 1972; Echols et al., 1973). This in turn prevents synthesis of the $c$ II and $c$ III gene products which are needed to activate synthesis of the $c$ I repressor (Echols \& Green, 1971; Reichardt \& Kaiser, 1971). Thus the cro gene product acts indirectly to inhibit synthesis of the $c I$ repressor. The cro gene product may also act directly to inactivate the cI repressor; however, the evidence on this point is equirocal. (A review of these data is presented by Echols (1972).)

One important difference between the cro and ant loci is that the cro gene function is required for lytic growth of $\lambda$, but ant function is not required for lytic growth of P22. Another difference between the cro and ant genes is their locations of the respective linkage maps. The cro gene lies between the $c \mathrm{~T}$ gene and the genes controlling DNA synthesis on the $\lambda$ map, while the ant gene lies far from the analogous position on the P22 map.

Another possible function of the ant gene product is interference with the activity of the $c 2$ repressor. For example, the antirepressor might compete with the $c 2$ repressor for binding sites on the phage genome, or it might inactivate the $c 2$ repressor by modifying it or by binding to it. Membrane filter binding experiments are now in progress (M. Gough, personal communication; M. Susskind \& D. Botstein, personal communication) to determine whether the ant gene product prevents binding of the c2 repressor to P22 DNA.

Other modes of action of the ant gene product are also possible. The ant gene product may not interact with the $c 2$ repressor at all, but rather overcomes $c 2$ repression indirectly. For example, the ant gene product might interact with RNA polymerase resulting in transcription of essential genes from promoter sites unaffected by the $c 2$ repressor. Another possibility is that the ant gene product may stimulate phage DNA synthesis causing titration of the $c 2$ repressor and subsequent progeny production.

At the moment we cannot distinguish among tho possible meohanisms of action of the ant gene product.

\section{(d) Concluding remarks}

It should be stressed that the immI region, as defined by the three loci, mnt virA and $a n t$, is dispensible both for replication and lysogeny. Double mutants of the $m n t$ 
ant and virA ant types (and presumably the triple mutant, mnt virA ant), in which the regulatory and structural gene functions are inactivated, are still viable temperate phages. They grow normally in sensitive cells, lysogenize with normal frequencies, give rise to stable prophage which are inducible by a variety of treatments and confer immunity to superinfection by homoimmune (albeit ant mutant) phages. In these mutants, the $i m m \mathrm{C}$ region is sufficient to impart all the characteristics of temperateness and lysogeny.

The dispensibility of the immI region for control of lysogeny raises the question of the selective advantage of these loci to a temperate phage. Genetic exchange may be enhanced between related temperate phages if the superinfecting phage not only replicates on a heteroimmune lysogen but also induces replication of the resident prophage. A demonstration that the ant gene product has broad spectrum antirepressor activity, that it can antagonize the $c$ repressor of many temperate phages, would make this an interesting suggestion. The $m n t$ repressor would then provide initial protection against antirepressor in homoimmune superinfection.

Phage P22 appears to be unusual among the temperate phages in controlling lysogeny and superinfection immunity through two separate and distantly linked gene clusters. The regulatory interactions of the loci and gene products of these clusters offer a new example of a complicated control apparatus, more complex than that of a single operon. The indication that the temperate phage Pl may also have the elements of a dual system for control of lysogeny suggests a wider applicability than for phage P22 alone (J. Scott, personal communication).

We would like to thank Mr Joseph Bellestri for his expert technical assistance, Dr Devid Botstein for several phage and bacterial strains, and Mr Steve Weaver, Drs Barbara Hoffman, Gail Wertz, David Friedman and especially Dr Ethel Jackson for valuable discussions and criticism of the manuscript.

This work was supported by Public Health Service grant GM-15419-07 from the National Institute for General Medical Sciences. One of us (S. T.) was supported by an American Cancer Society Postdoctoral Fellowship (no. PF790). Another author (M. J. B.) was a National Institutes of Health predoctoral fellow supported by Training grant GM-00071 from the U.S. Public Health Service.

\section{REFERENCES}

Bezdek, M. \& Amati, P. (1968). Virology, 36, 701-703.

Botstein, D., Lew, K. K., Jarvik, V. \& Swanson, C. A. Jr (1975). J. Mol. Biol. 91, 439-462.

Bronson, M. \& Levine, M. (1971). J. Virol. 7, 559-568.

Bronson, M. \& Levine, M. (1972). Virology, 47, 644-655.

Chan, R. K. \& Botstein, D. (1972). Virology, 49, 257-267.

Court, D. \& Campbell, A. (1972). J. Virol. 9, 938-945.

Echols, H. (1972). Annu. Rev. Genet. 6, 157-190.

Echols, H. \& Green, L. (1971). Proc. Nat. Acad. Sci., U.S.A. 68, 2190-2194.

Echols, H., Green, L., Oppenheim, A. B., Oppenheim, A. \& Honigman, A. (1973). J. Mol. Biol. 80, 203-216.

Gough, M. (1968). J. Virol. 2, 992-998.

Levine, M. (1957). Virology, 3, 22-41.

Levine, M. (1972). Current Topics in Microbiol. and Immunol. 58, 135-156.

Levine, M., Chakravorty, M. \& Bronson, M. J. (1970). J. Virol. 6, 400-405.

Ptashne, M. (1971). In The Bacteriophage Lambda (Hershey, A. D., ed.), pp. 221-237, Cold Spring Harbor Laboratory, New York.

Rao, R. N. (1968). J, Mol. Biol. 35, 607-622. 
Reichardt, L. \& Kaiser, A. D. (1972). Proc. Nat. Acad. Sci., U.S.A. 68, 2185-2189. Smith, H. O. \& Levine, M. (1967). Virology, 31, 207-216.

Susskind, M., Wright, A. \& Botstein, D. (1971). Virology, 45, 638-652.

Walsh, J. \& Meynell, G. G. (1967). J. Gen. Virology, 1, 581-582.

Zinder, N. D. (1958). Virology, 5, 291-326. 